\title{
A Hepatic Cyst Complicated by Hemorrhage with Secondary Revascularization Mimicking Mucin-Producing Cystic Neoplasm: Radiologic-Pathologic Correlations
}

\author{
Ji Soo Song, Jae Do Yang, ${ }^{2}$ and Woo Sung Moon ${ }^{3, *}$ \\ ${ }^{1}$ Department of Radiology, Chonbuk National University Medical School and Hospital, Research Institute of Clinical Medicine of \\ Chonbuk National University, Biomedical Research Institute of Chonbuk National University Hospital, Jeonju, Korea \\ ${ }^{2}$ Department of Surgery, Chonbuk National University Medical School and Hospital, Jeonju, Korea \\ ${ }^{3}$ Department of Pathology, Chonbuk National University Medical School, and Research Institute for Endocrine Sciences, Jeonju, Korea \\ "Corresponding author: Woo Sung Moon, Department of Pathology, Chonbuk National University Medical School, and Research \\ Institute for Endocrine Sciences, 20 Geonji-ro, Doekjin-gu, Jeonju, Chonbuk, 54907, Korea. Tel: +82-632703086, Fax: +82-632703135, \\ E-mail: mws@chonbuk.ac.kr
}

Received 2017 May 11; Revised 2017 July 31; Accepted 2018 January 23.

\begin{abstract}
Cystic hepatic lesions are commonly encountered in clinical practice. Most of them are simple hepatic cysts such as biliary cysts. However, complicated biliary cysts with hemorrhage, necrosis, and organization pose diagnostic challenges since imaging findings overlap with those of neoplastic cystic lesions. Here, we report a case of biliary cyst complicated by hemorrhage with secondary revascularization with ultrasound, computed tomography, and gadoxetic acid-enhanced magnetic resonance imaging findings mimicking mucin-producing cystic neoplasm.
\end{abstract}

Keywords: Biliary Cyst, Complicated Cyst, Revascularization, Organization, Mucinous Cystic Neoplasm

\section{Introduction}

Cystic hepatic lesions, including developmental, inflammatory, neoplastic, and trauma-related lesions, are commonly encountered in daily clinical practice. Most cystic hepatic lesions have classic imaging findings, and diagnoses are often made based solely on imaging findings. However, neoplastic cystic hepatic lesions such as mucinous cystic neoplasm (MCN), cystic hemangioma, cystic hepatocellular carcinoma, cystic metastasis, and cystforming intraductal papillary neoplasm of the bile duct (IPNB), which are rarely encountered in clinical practice, pose diagnostic challenges (1-3). In addition, complicated biliary cysts (e.g., hemorrhage, necrosis, septation, and secondary revascularization during the process of organization) can be misdiagnosed as neoplastic cystic hepatic lesions. The differential diagnosis of these neoplastic and non-neoplastic lesions is important since treatments and prognoses vary among them.

Here, we present a complex cystic hepatic lesion case pathologically shown to be a biliary cyst complicated by hemorrhage with secondary revascularization using imaging to detect cyst-forming IPNB or MCN.

\section{Case Presentation}

A 79-year-old male patient was referred to our hospital for management of an incidentally-detected liver mass on health screening ultrasonography. The patient was receiving medication for underlying diabetes and hypertension. His physical examination and laboratory findings were unremarkable. Tumor markers such as alphafetoprotein, carcinoembryonic antigen, and carbohydrate antigen 19 - 9 (CA 19-9) were within normal limits. Serological tests for hepatitis $\mathrm{B}$ and $\mathrm{C}$ were negative. Abdominal ultrasonography demonstrated a 7-cm multilocular cystic mass with heterogeneous inner echogenicity and posterior acoustic enhancement in the posterior section of the right hepatic lobe (Figure 1). On four-phase dynamic computed tomography (CT), the multilocular cystic mass showed avid enhanced foci on the periphery, with these foci showing progressive and persistent enhancement on 
subsequent portal venous and delayed phases (Figure 2). On gadoxetic acid-enhanced magnetic resonance imaging (MRI), the mass showed bright high signal intensity (SI) on T2 weighted images (WI), low SI on T1WI, the same enhancement pattern on dynamic phase imaging, and no areas of diffusion restriction on diffusion weighted imaging. On 20-minute hepatobiliary phase (HBP) imaging, the mass demonstrated subtle high SI compared with dynamic phase imaging due to contrast uptake (Figure 3). The imaging-based differential diagnosis included cystforming IPNB with invasive carcinoma and MCN with invasive carcinoma. The patient underwent right posterior sectionectomy. The surgical specimen revealed a welldemarcated cystic mass filled with a chocolate-colored hematoma and multiple dilated bile ducts (Figure 4A). Histologically, the lesion was well defined from the hepatic tissue by a fibrous wall. Most of this lesion was composed of a blood clot that was partly liquefied (Figure 4B). The remaining small solid portion was a proliferation of capillarysized vessels and several large vessels with a muscular wall, suggesting secondary revascularization during the process of organizing the hematoma (Figure 4C). The epithelia lining large cyst walls were denuded; however, the adjacent intact cystic lesions were lined with simple cuboidal biliary epithelium (Figure 4D). Neither a tumorous component nor ovarian-type stroma was found. The final diagnosis was biliary cyst complicated by hemorrhage with secondary revascularization. The postoperative course was uneventful. Nine months later, the patient remained in good condition without any complaints.

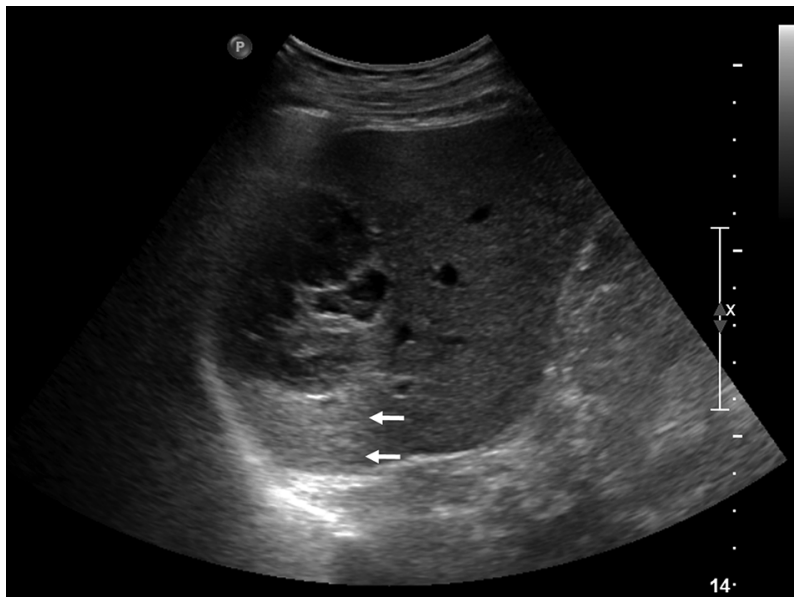

Figure 1. Ultrasound image demonstrates a $7 \mathrm{~cm}$ multilocular cystic mass in the posterior section of the right hepatic lobe with heterogeneous inner echogenicity. The presence of posterior acoustic enhancement (arrows) reflects the cystic nature of the mass.

\section{Discussion}

Mucin-producing cystic neoplasms of the liver consist of MCN and cyst-forming IPNB, which are considered premalignant or malignant lesions with similar macroscopic features (2). MCN includes biliary cystadenoma (BCA) and biliary cystadenocarcinoma (BCAC), and is defined as a cyst-forming epithelial neoplasm composed of mucin-producing epithelium and ovarian-type stroma (1). Both lesions are usually multilocular with enhanced walls, multiple septa, and variable degrees of calcification (4). Enhanced mural nodules are more commonly encountered in BCAC than BCA (5). Cyst-forming IPNB is a subtype of IPNB, which was previously categorized as biliary papilloma and papillomatosis (6). Cyst-forming IPNB lacks ovarian-type stroma and communicates with the bile duct, unlike MCN (2). However, the imaging findings for cystforming IPNB are similar to those of MCN, except for the presence of a mural nodule, and upstream and downstream biliary dilatation. Communication between hepatic cystic lesions and the bile duct is more common in cyst-forming IPNB (7). A recent study using gadoxetic acid-enhanced MRI demonstrated contrast uptake on 20minute HBP imaging in cyst-forming IPNB, reflecting the presence of communication between the lesion and the bile duct (8). Our case showed a mural nodule and contrast uptake on HBP imaging, which led to cyst-forming IPNB as our first differential diagnosis.

Although rare, differentiating biliary cysts and mucinproducing cystic neoplasms can pose diagnostic challenges, as in our case. Biliary cysts usually do not require treatment unless symptomatic, while mucin-producing cystic neoplasms are treated with surgical resection (9). Thus, differentiation of these lesions is important. Kim et al. reported that the presence of septa, central septa, mural nodule, upstream bile duct dilatation, and downstream bile duct dilatation were significant CT findings differentiating mucin-producing cystic neoplasms from solitary bile duct cysts ( $\mathrm{P}<0.05$ for each finding) (7). Septa were present in both lesions but the incidence was significantly higher in mucin-producing cystic neoplasms ( $\mathrm{P}$ $=0.001)$. Mural nodules $(\mathrm{P}<0.001)$, mosaic pattern $(\mathrm{P}=$ $0.113)$, and communication with the bile duct $(\mathrm{P}=0.053)$ were only present in mucin-producing cystic neoplasms. They concluded that when CT findings were used in combination, mucin-producing cystic neoplasms could be differentiated from biliary cysts with a high degree of diagnostic accuracy (7). In our case, the mass showed septa and mural nodules (which later proved to be congeries of small capillaries due to secondary revascularization in the process of organization), without central septa or biliary dilatation. Since upstream and downstream biliary dilata- 

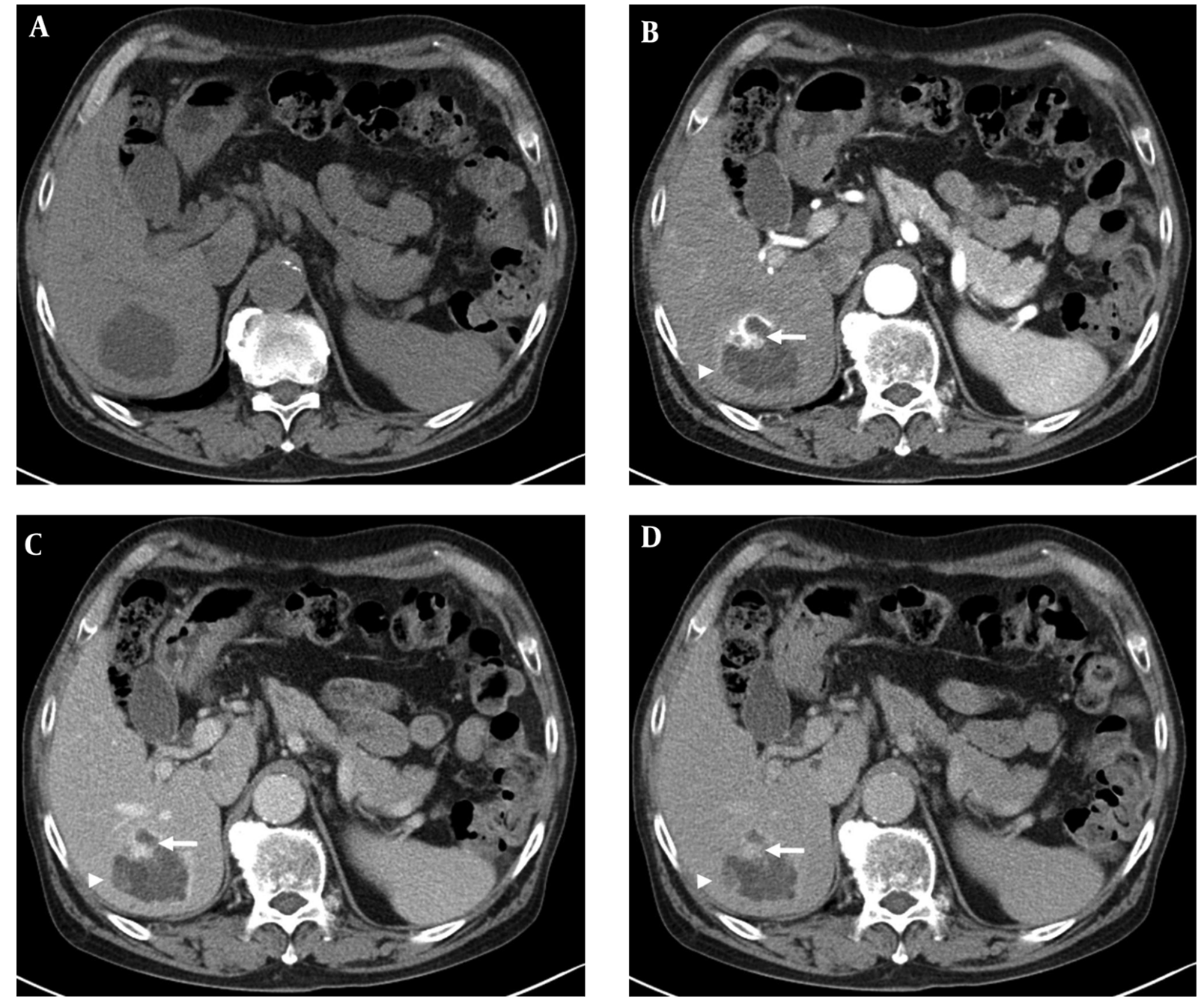

Figure 2. A, Unenhanced CT image shows a $7 \mathrm{~cm}$ cystic mass with heterogeneous low attenuation and a minimally lobulated contour. B, Arterial phase CT image shows an avidly enhanced portion in the periphery of the cystic mass (arrow). C, Portal and D, Delayed phase CT images show progressive and persistent enhancement of the previously avid enhanced portion in B. There is another progressive enhancing nodular lesion in the periphery (arrowheads in B, C, and D).

tion are highly specific (90\% - 100\% specificity) findings for mucin-producing cystic neoplasms, biliary cyst should have been considered during the differential diagnosis in the present case (7).

Based on the clinical and imaging findings, the first differential diagnosis for our patient was cyst-forming IPNB with invasive carcinoma. Even though the patient was a 79year-old male, the second differential diagnosis was MCN with invasive carcinoma due to the multilocular cystic appearance of the mass with enhanced walls. There were strong enhanced portions in the periphery of the cystic mass, and these lesions showed prolonged enhancement. This finding was regarded as indicating an invasive carcinoma arising from an underlying mucin-producing cys- tic neoplasm. However, upon radiologic-pathologic correlation, the organizing tissue component composed of large muscular vessels surrounded by congeries of small capillaries was compatible with the enhanced portions on imaging studies. In addition, the multilocular cystic appearance on CT and MRI proved to indicate small capillary linings around an organized hematoma, not true septa or multilocular cysts. On 20-minute HBP imaging, the mass exhibited higher SI than on dynamic phase imaging. This could be interpreted as indicating either bile duct communication within the mass or extracellular contrast agent pooling due to excessive fibrosis (10). Gadoxetic acid is a widely-used hepatocyte-specific MR contrast agent, which has characteristics of both extracellular and 

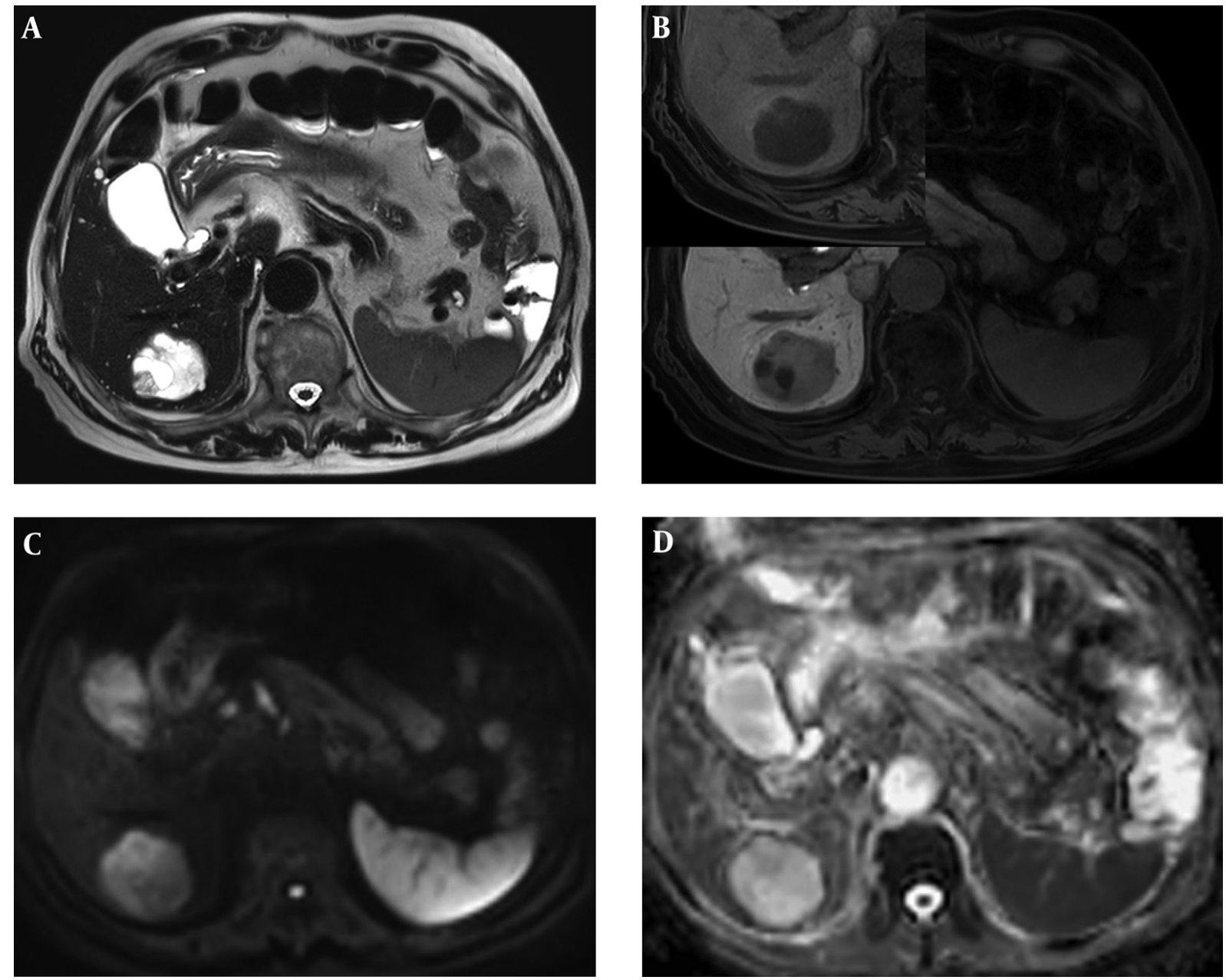

Figure 3. A, Heavily weighted T2 image [echo time $(\mathrm{TE})=160$ ] shows heterogeneous high signal intensity of a multilocular cystic mass with a minimally lobulated contour. $\mathrm{B}, 20$-minute hepatobiliary phase imaging shows mass contrast uptake due to contrast excretion into bile duct and the presence of biliary communication within the mass. Inset Unenhanced T1 weighted image for comparison. C, B $=800 \mathrm{~s} / \mathrm{mm}^{2}$ and $\mathrm{D}$, Apparent diffusion coefficient image demonstrates no areas of diffusion restriction.

liver-specific contrast agents with approximately $50 \%$ of the intravenously injected gadoxetic acid being excreted via the bile duct (11). When a lesion shows contrast uptake on 20-minute HBP imaging, there are two possible mechanisms: 1) the presence of functioning hepatocytes with biliary excretion and/or biliary communication enabling contrast uptake, or 2) the presence of severe fibrosis with abundant extracellular space causing pooling of the contrast agent $(12,13)$. However, the latter mechanism is usually seen in solid tumors such as intrahepatic massforming type cholangiocarcinomas (14). On gross pathologic findings, our case revealed that the bile duct was inside the mass, compatible with bile duct communication, and this explained the lesion's contrast uptake. Most recently, Ying et al. showed that gadoxetic acid revealed an intraductal mucin component and biliary communication in IPNB (8). We hypothesize that gadoxetic acid-enhanced MRI may be a promising imaging modality for the diagnosis of biliary communication within cystic lesions, although additional study is warranted.

On diffusion weighted imaging, the mass revealed no areas of diffusion restriction. Several recent studies have shown that diffusion restriction could be useful for intraductal IPNB solid component detection and tumor invasiveness determination (15-17). Based on retrospective findings, mismatch between the prolonged enhanced portion and the absence of diffusion restriction could be an important clue for differential diagnosis of biliary cysts with hemorrhage and revascularization, rather than invasive carcinoma, in mucin-producing cystic neoplasms. How- 

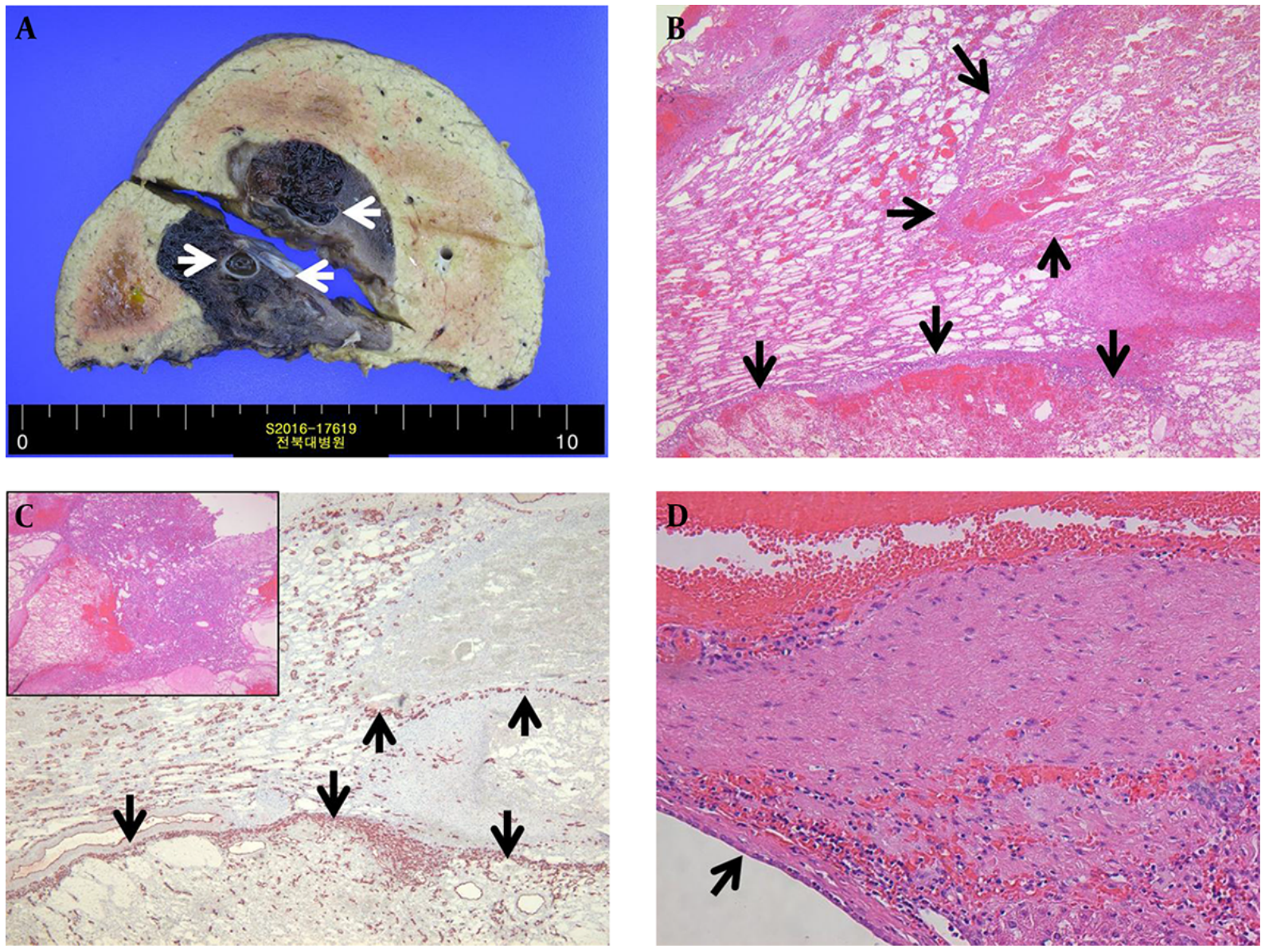

Figure 4. A, A well-demarcated cystic mass filled with chocolate-colored hematoma containing multiple hemorrhagic biliary cysts (arrows). B, Most of this lesion was composed of a blood clot that was partly liquefied. The proliferation of capillary-sized vessels in the peripheral portion of the hemorrhage caused a septate, lobular appearance (arrows). C, Endothelial cell marker CD34 immunostaining highlighted the proliferating capillary vessels (arrows). Inset The solid portion of the lesion was composed of proliferating capillary-sized vessels and several large vessels with muscular walls. D, The cystic lesion was lined with simple cuboidal biliary epithelium (arrow), consistent with a biliary cyst.

ever, the diagnostic value of diffusion weighted imaging for differentiating mucin-producing cystic neoplasms and biliary cysts requires validation by additional studies.

In conclusion, we presented a biliary cyst case complicated by hemorrhage with secondary revascularization in an elderly male patient. Gadoxetic acid-enhanced MRI could be a useful imaging modality for detecting bile duct communication within a cystic lesion. When a complex cystic mass with a persistent enhanced solid portion is detected, although rare, the possibility of complicated biliary cyst with secondary revascularization should be included in the differential diagnosis in addition to mucinproducing cystic neoplasms.

\section{Acknowledgments}

This work was supported by the national research foundation of Korea (NRF) grant funded by the Korean government (MSIP) (No. 2008-0062279).

\section{Footnotes}

Authors' Contributions: Ji Soo Song wrote the manuscript. Jae Do Yang served as an attending doctor for the patient, and performed the surgery. Woo Sung Moon performed the pathological examination. Jae Do Yang and Woo Sung Moon edited the manuscript.

Conflicts of Interest: None. 


\section{References}

1. Devaney K, Goodman ZD, Ishak KG. Hepatobiliary cystadenoma and cystadenocarcinoma. A light microscopic and immunohistochemical study of 70 patients. Am J Surg Pathol.1994;18(11):1078-91. [PubMed: 7943529].

2. Theise N, Nakashima O, Park Y, Nakanuma Y, Bosman F, Garneiro F. Hepatocellular Carcinoma. WHO classification of tumours of the digestive system. In: Bosman FT, Carneiro F, Hruban RH, editors. Lyon: IARC Press; 2010. p. 205-16.

3. Wheeler DA, Edmondson HA. Cystadenoma with mesenchymal stroma (CMS) in the liver and bile ducts. A clinicopathologic study of 17 cases, 4 with malignant change. Cancer. 1985;56(6):1434-45. [PubMed: 4027877].

4. Buetow PC, Buck JL, Pantongrag-Brown L, Ros PR, Devaney K, Goodman ZD, et al. Biliary cystadenoma and cystadenocarcinoma: clinicalimaging-pathologic correlations with emphasis on the importance of ovarian stroma. Radiology. 1995;196(3):805-10. doi: 10.1148/radiology.196.3.7644647. [PubMed: 7644647].

5. Kim JY, Kim SH, Eun HW, Lee MW, Lee JY, Han JK, et al. Differentiation between biliary cystic neoplasms and simple cysts of the liver: accuracy of CT. AJR Am J Roentgenol. 2010;195(5):1142-8. doi: 10.2214/AJR.09.4026. [PubMed: 20966320].

6. Lim JH, Zen Y, Jang KT, Kim YK, Nakanuma Y. Cyst-forming intraductal papillary neoplasm of the bile ducts: description of imaging and pathologic aspects. AJR Am J Roentgenol. 2011;197(5):1111-20. doi: 10.2214/AJR.10.6363. [PubMed: 22021503].

7. Kim HJ, Yu ES, Byun JH, Hong SM, Kim KW, Lee JS, et al. CT differentiation of mucin-producing cystic neoplasms of the liver from solitary bile duct cysts. AJR Am J Roentgenol. 2014;202(1):83-91. doi: 10.2214/AJR.12.9170. [PubMed: 24370132].

8. Ying $\mathrm{SH}$, Teng XD, Wang ZM, Wang QD, Zhao YL, Chen F, et al. Gd-EOB-DTPA-enhanced magnetic resonance imaging for bile duct intraductal papillary mucinous neoplasms. World J Gastroen- terol. 2015;21(25):7824-33. doi: 10.3748/wjg.v21.i25.7824. [PubMed: 26167082].

9. Emre A, Serin KR, Ozden I, Tekant Y, Bilge O, Alper A. Intrahepatic biliary cystic neoplasms: Surgical results of 9 patients and literature review. World J Gastroenterol. 2011;17(3):361-5. [PubMed: 26167082].

10. Choi JY, Lee JM, Sirlin CB. CT and MR imaging diagnosis and staging of hepatocellular carcinoma: part II. Extracellular agents, hepatobiliary agents, and ancillary imaging features. Radiology. 2014;273(1):30-50. doi: 10.1148/radiol.14132362. [PubMed: 25247563].

11. Higaki A, Tamada T, Sone T, Kanki A, Sato T, Tanimoto D, et al. Potential clinical factors affecting hepatobiliary enhancement at Gd-EOB-DTPAenhanced MR imaging. Magn Reson Imaging. 2012;30(5):689-93. doi: 10.1016/j.mri.2012.01.004. [PubMed: 22459437].

12. Vanderveen KA, Hussain HK. Magnetic Resonance Imaging of cholan giocarcinoma. Cancer Imaging. 2004;4(2):104-15. doi: 10.1102/14707330.2004.0018. [PubMed: 18250017].

13. Sari S, Akgun V, Battal B, Karaman B. Re: communication between the cystic lesions of the liver and biliary tree: how can we evaluate efficiently and safely? Korean J Radiol. 2014;15(4):540. doi: 10.3348/kjr.2014.15.4.540. [PubMed: 25053917].

14. Kang Y, Lee JM, Kim SH, Han JK, Choi BI. Intrahepatic mass-forming cholangiocarcinoma: enhancement patterns on gadoxetic acidenhanced MR images. Radiology. 2012;264(3):751-60. doi: 10.1148/radiol.12112308. [PubMed: 22798225].

15. Yoon HJ, Kim YK, Jang KT, Lee KT, Lee JK, Choi DW, et al. Intraductal papillary neoplasm of the bile ducts: description of MRI and added value of diffusion-weighted MRI. Abdom Imaging. 2013;38(5):1082-90. doi: 10.1007/s00261-013-9989-4. [PubMed: 23508835].

16. Cui XY. Role of diffusion-weighted magnetic resonance imaging in the diagnosis of extrahepatic cholangiocarcinoma. World J Gastroenterol. 2010;16(25):3196. doi: 10.3748/wjg.v16.i25.3196.

17. Joo I, Lee JM. Imaging bile duct tumors: pathologic concepts, classification, and early tumor detection. Abdom Imaging. 2013;38(6):1334-50. doi: 10.1007/s00261-013-0027-3. [PubMed: 23925840]. 